\title{
Role of Gamma Knife surgery in patients with 5 or more brain metastases
}

\author{
Clinical article
}

\author{
Alireza Mohammad Mohammadi, M.D.,${ }^{1-3}$ Pablo F. Recinos, M.D. ${ }^{1-3}$ \\ Gene H. BarnetT, M.D., M.B.A. ${ }^{1-3,5}$ RoberT J. WeIL, M.D., ${ }^{1-3,5}$ \\ Michael A. Vogelbaum, M.D., Ph.D., ${ }^{1-3,5}$ Samuel T. Chao, M.D., ${ }^{1,4,5}$ John H. Suh, M.D., ${ }^{1,4,5}$ \\ Nicholas F. Marko, M.D. ${ }^{7}$ Paul Elson, Sc.D., 6 Gennady Neyman, Ph.D., 4,5 \\ and Lilyana Angelov, M.D. ${ }^{1-3,5}$
}

${ }^{I}$ The Rose Ella Burkhardt Brain Tumor and Neuro-Oncology Center, ${ }^{2}$ Department of Neurosurgery; ${ }^{3}$ Neurological Institute; ${ }^{4}$ Department of Radiation Oncology; ${ }^{5}$ Taussig Cancer Institute; and ${ }^{6}$ Department of Quantitative Health Science, Cleveland Clinic, Cleveland, Ohio; and ${ }^{7}$ Department of Applied Mathematics and Theoretical Physics \& Cancer Research, UK Cambridge Research Institute, Cambridge University, Cambridge, United Kingdom

Object. The authors evaluated overall survival and factors predicting outcome in patients with $\geq 5$ brain metastases who were treated with Gamma Knife surgery (GKS).

Methods. Medical records from patients with $\geq 5$ brain metastases treated with GKS between 1997 and 2010 at the Cleveland Clinic Gamma Knife Center were retrospectively reviewed. Patient demographics, tumor characteristics, treatment-related factors, and outcome data were evaluated.

Results. One hundred seventy patients were identified, with a median age of 58 years. The female/male ratio was 1.2:1. Gamma Knife surgery was used as an upfront treatment in $35 \%$ of patients and as salvage treatment in $65 \%$ of patients with multiple brain metastases. The median overall survival after GKS was 6.7 months (95\% CI 5.5-8.1). At the time of GKS, 128 patients (75\%) had concurrent extracranial metastases, and in 69 patients (41\%) multiple extracranial sites were involved. Ninety-two patients (54\%) had a history of whole-brain radiation therapy, and 158 patients (93\%) had a Karnofsky Performance Scale (KPS) score $\geq 70$. The median total intracranial disease volume was $3.2 \mathrm{~cm}^{3}$ (range $0.2-37.2 \mathrm{~cm}^{3}$ ). A total intracranial tumor volume $\geq 10 \mathrm{~cm}^{3}$ was observed in 32 patients $(19 \%)$. Lower KPS score at the time of treatment $(p<0.0001)$, patient age $>60$ years $(p=0.004)$, multiple extracranial metastases $(p=0.0001)$, and greater intracranial burden of disease $(p=0.03)$ were prognostic factors for poor outcome in the univariate and multivariate analyses.

Conclusions. In this study, GKS was safe and effective for upfront and salvage treatment in patients with $\geq 5$ brain metastases. Gamma Knife surgery should be considered as an additional treatment modality for these patients, especially in the subset of patients with favorable prognostic factors.

(http://thejns.org/doi/abs/10.3171/2012.8.GKS12983)

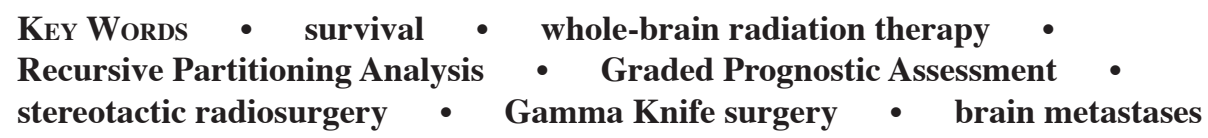

$\mathrm{B}$ RAIN metastases develop in $20 \%-40 \%$ of patients with systemic cancer and are a major cause of morbidity and mortality in these patients. ${ }^{2,24-26,34}$ The incidence appears to be increasing due to improved

Abbreviations used in this paper: GKS = Gamma Knife surgery; GPA = Graded Prognostic Assessment; KPS = Karnofsky Performance Scale; RCT = randomized clinical trial; RPA = Recursive Partitioning Analysis; SRS = stereotactic radiosurgery; $\mathrm{WBRT}=$ whole-brain radiation therapy. neuroimaging technology and improved systemic disease therapies. These advances have resulted in an earlier diagnosis and longer survival from the primary cancer $^{14,35,37}$ Whole-brain radiation therapy is one modality used to treat brain metastases and is currently the primary modality used for patients with $\geq 5$ metastases. ${ }^{6,9,27,39,40,44}$ Gamma Knife surgery is a standard treatment modality for patients with a single or a limited number $(\leq 4)$ of brain metastasis; however, its role in the treatment of patients with $\geq 5$ metastases is less well defined. 2,3,7,20 
For patients with a limited number of brain metastases ( $\leq 4$ lesions), there is Level I evidence that the addition of radiosurgery to WBRT increases overall survival and results in improved local tumor control.,42 In addition, radiosurgery alone has been shown to produce similar survival and less cognitive decline than radiosurgery plus WBRT, albeit with lower local and distant control rates of intracranial disease. ${ }^{3,7,20}$ The most important prognostic factors for patients with $\leq 4$ metastases are KPS score, age, extent of extracranial metastatic disease, extent of systemic disease control, number of intracranial lesions, and intracranial tumor burden. ${ }^{2,11,13,23,33}$ Recently, there has been increasing interest in offering GKS to patients with a more extensive intracranial disease burden $(\geq 5$ metastases) as an upfront treatment or in combination with WBRT.,17,31 There are a limited number of studies that have evaluated the outcomes of patients with $\geq 5$ brain metastases treated with GKS. In most retrospective series, a higher number of metastatic lesions has not been shown to change survival for patients with $\geq 5$ brain metastases. ${ }^{4,8,18,29}$ In addition, our initial analysis in a smaller group of patients with $\geq 5$ metastases (64 patients) determined that a lower KPS score was predictive of survival and was suggestive that GKS may also have a role in treating this group of patients in addition to WBRT. ${ }^{16}$

In this study, we evaluated the impact of GKS treatment on overall survival in patients with $\geq 5$ brain metastases. To our knowledge, this is the largest reported series in which this group has been evaluated. We also examined associated factors to determine their effect on patient outcomes.

\section{Methods}

A retrospective review of patients with $\geq 5$ brain metastases treated with GKS at the Cleveland Clinic Gamma Knife Center between 1997 and 2010 was performed. Patients were included if they were $\geq 18$ years old and had $\geq 5$ metastatic brain lesions treated with GKS during a single session. Patients who had no clinical or imaging follow-up after GKS were excluded.

\section{Patient Characteristics}

One hundred seventy patients were identified as having met the inclusion criteria. The median age of these patients was 58 years (range 18-81 years) at the time of treatment with GKS. There were 94 female $(55 \%)$ and 76 male $(45 \%)$ patients. The most frequent primary disease was lung cancer (49\%), followed by breast cancer $(20 \%)$ and melanoma (16\%). The median time between diagnosis of the primary tumor and neurological involvement was 1 year (range $0-18$ years). In 34 patients (20\%), concurrent brain metastases were diagnosed at the time of the primary diagnosis. The median interval from neurological involvement to treatment with GKS for $\geq 5$ lesions was 5.1 months (range 0-49 months). In 60 patients (35\%), the first neurological presentation was associated with $\geq 5$ brain metastases. There were 102 patients $(60 \%)$ who had a history of previous treatment for brain metastases (single treatment or combined therapies) before treatment with GKS for $\geq$ 5 brain metastases. In those patients who had undergone treatment earlier, the median interval between the most recent treatment and GKS for $\geq 5$ lesions was 6.2 months (range 1-48 months). In the months prior to GKS, 96 patients $(56 \%)$ received chemotherapy for active systemic disease. In more than half of the patients (56\%), there were no other systemic metastases at the time of the initial diagnosis of metastatic disease to the brain, whereas at the time of GKS treatment for $\geq 5$ lesions, $75 \%$ of patients had other extracranial metastases. The median KPS score in the patients was 80 (range 60-100). There were 25 RPA Class I (15\%), 135 RPA Class II (79\%), and 10 RPA Class III patients (6\%). For the 60 patients who were treated with upfront GKS for $\geq 5$ brain metastases, the RPA classification was as follows: 13 patients in RPA Class I (22\%), 45 patients in RPA Class II (75\%), and 2 patients in RPA Class III (3\%). Approximately half of the patients had a GPA score $>1$ (Table 1).

\section{Treatment Details}

During the study period, GKS was performed using Gamma Knife models B, C, 4C, and Perfexion (Elekta AB). Our standard protocol began with frame application after the patient had been administered a local anesthetic agent and an intravenous sedative/anxiolytic. After the frame was applied to the patient's head, high-resolution, contrast-enhanced MRI (if not clinically contraindicated) and CT scanning of the brain were performed in all patients. Treatment planning was performed on volumetric, contrast-enhanced MR images (1-mm slices) and was supplemented by contrast-enhanced T1-weighted imaging (2-mm slices). In all cases, MR-CT image matching was performed to enhance accuracy and eliminate possible distortions of MRI sequences. The Radiation Therapy Oncology Group 90-05 dosing protocol was used as the treatment guideline, and all lesions were treated at $50 \%$ or higher isodose lines. ${ }^{30}$ Patients had initial follow-up with a clinic visit and repeat MRI 4-8 weeks after GKS and subsequently every 3 months. This study was approved by the Cleveland Clinic Institutional Review Board.

\section{Statistical Analysis}

Patient characteristics were summarized using frequency counts and percentages for categorical factors and by using medians and ranges for continuous factors. Patients were also categorized by their RPA ${ }^{12}$ and GPA ${ }^{35}$ classes. For convenience, a recursive portioning algorithm was also used to find optimal cutoffs for maximum tumor diameter and tumor volume. Overall survival, measured from the date of GKS to the patient's death or last followup, was the primary outcome. Progression-free survival was a secondary outcome measure and was calculated from the time of GKS to the time of documented tumor recurrence (local or distant). Both outcomes were summarized using the Kaplan-Meier method. The log-rank test and proportional hazards model were used for univariate comparisons of overall survival. The proportional hazards model, in conjunction with a stepwise selection algorithm that used $\mathrm{p}=0.10$ and $\mathrm{p}=0.05$ as the criteria for entry and retention in the model, respectively, was 
TABLE 1: Characteristics in 170 patients with $\geq 5$ brain metastases and the results of the univariate survival analysis

\begin{tabular}{|c|c|c|c|}
\hline Factor & No. of Patients (\%) & Median Survival (mos) & p Value \\
\hline \multicolumn{4}{|l|}{ patient sex } \\
\hline female & $94(55)$ & 6.7 & \\
\hline male & $76(45)$ & 6.1 & 0.07 \\
\hline \multicolumn{4}{|l|}{ patient age (yrs) } \\
\hline$<60$ & $108(64)$ & 8.1 & \\
\hline$>60$ & $62(36)$ & 4.2 & $<0.0001$ \\
\hline \multicolumn{4}{|l|}{ primary disease } \\
\hline lung cancer & $83(49)$ & 7.0 & \\
\hline breast cancer & $34(20)$ & 9.1 & \\
\hline melanoma & $28(16)$ & 4.7 & \\
\hline renal cell carcinoma & $16(9)$ & 7.0 & \\
\hline other & $9(5)$ & 3.0 & 0.1 \\
\hline \multicolumn{4}{|l|}{ prior treatments } \\
\hline any & $108(64)$ & 6.8 & 0.5 \\
\hline WBRT (total) & $92(54)$ & 7.1 & 0.5 \\
\hline SRS (total) & $38(22)$ & 6.7 & 0.6 \\
\hline surgery (total) & $29(17)$ & 8.2 & 0.2 \\
\hline \multicolumn{4}{|l|}{ extracranial metastases } \\
\hline none & $42(25)$ & 9.5 & \\
\hline single & $59(35)$ & 7.4 & \\
\hline multiple & $69(41)$ & 5.0 & 0.006 \\
\hline \multicolumn{4}{|l|}{ KPS score } \\
\hline 60 & $12(7)$ & 1.4 & \\
\hline 70 & $37(22)$ & 4.7 & \\
\hline 80 & $55(32)$ & 6.1 & \\
\hline $90-100$ & $66(39)$ & 9.7 & $<0.0001$ \\
\hline \multicolumn{4}{|l|}{ RPA class } \\
\hline 1 & $25(15)$ & 6.7 & \\
\hline II & $135(79)$ & 7.0 & \\
\hline III & $10(6)$ & 1.7 & 0.01 \\
\hline \multicolumn{4}{|l|}{ GPA score } \\
\hline $0-1$ & $84(49)$ & 4.7 & \\
\hline $1.5-3.5$ & $86(51)$ & 9.5 & 0.0004 \\
\hline \multicolumn{4}{|l|}{ neurological impairment } \\
\hline none & $34(20)$ & 8.3 & \\
\hline mild & $107(63)$ & 7.0 & \\
\hline moderate/severe & $29(17)$ & 3.4 & 0.0006 \\
\hline \multicolumn{4}{|l|}{ total intracranial tumor vol } \\
\hline$<1 \mathrm{~cm}^{3}$ & $35(21)$ & 10.6 & \\
\hline $1-10 \mathrm{~cm}^{3}$ & $103(60)$ & 6.9 & \\
\hline$>10 \mathrm{~cm}^{3}$ & $32(19)$ & 4.1 & 0.001 \\
\hline
\end{tabular}

also used in a multivariate analysis to identify independent prognostic factors. Data analyses were performed using SAS version 9.2 (SAS Inc.).

\section{Results}

In patients with $\geq 5$ brain metastases, GKS was used as the sole upfront treatment in 32 patients (19\%), as a boost to upfront WBRT in 28 patients $(16 \%)$, and as salvage treatment in 110 patients (65\%). Whole-brain radiation therapy had been performed previously in 92 patients (54\%). Of those patients who had received WBRT, 22 patients (13\%) received both GKS and WBRT. In addition, in 16 patients (9\%) GKS was the sole treatment. The median number of targets treated at each GKS session was 6 (range 5-20 targets), and a total of 1101 metastatic brain lesions were 
treated. In 105 patients (62\%) both supra- and infratentorial lesions were observed; 63 patients $(37 \%)$ had only supratentorial lesions and 2 patients $(1 \%)$ had only infratentorial lesions. Brainstem metastases were observed in 24 patients in whom there was infratentorial involvement (14\%). Eight hundred sixty-seven supratentorial tumors (79\% of patients; maximum 13 lesions per patient) and 234 infratentorial tumors ( $21 \%$ of patients; maximum 8 lesions per patient) were treated with GKS. The median total intracranial tumor burden was $3.2 \mathrm{~cm}^{3}$ (range $0.2-37.2 \mathrm{~cm}^{3}$ ) and the median maximum dimension of the largest treated target was $1.8 \mathrm{~cm}$ (range $0.5-5.1 \mathrm{~cm}$ ).

\section{Patient Outcomes}

The median duration of follow-up was 6.2 months after GKS for $\geq 5$ lesions (mean 7.2 months, range 1-56 months), and 148 patients (87\%) died during the follow-up period. Neurological disease progression was the primary cause of death in 39 patients (26\%), and systemic disease progression was the cause of death in 57 patients (39\%). Notably, in $35 \%$ of patients, the cause of death could not be determined due to incomplete chart documentation and/or the lack of recent imaging studies. As our center is a tertiary referral center, some patients return to their local hospitals for oncological treatment and the cause of death in these patients is difficult to determine. The median survival times were 29 months (range 1-237 months) after diagnosis of the primary tumor, 13 months (range 1-106 months) after neurological involvement, and 6.7 months (range 1-66 months) after GKS for $\geq 5$ lesions. The median survival times were 6.4 months after upfront GKS, 6.5 months after GKS was added to upfront WBRT, and 6.8 months after salvage GKS ( $p>0.05)$. At the time of the last follow-up, 22 patients were still alive with controlled intracranial disease (median follow-up 5.4 months). Estimated 6-month and 1-year survival rates were $56 \%$ and $26 \%$, respectively.

Imaging-verified disease progression occurred in 87 patients (51\%) during the follow-up period. Isolated local recurrences occurred in 6 patients (3\%), local and distant recurrences occurred in 13 patients $(8 \%)$, and isolated distant recurrences occurred in 68 patients (40\%). One $(17 \%)$ of 6 patients with isolated local tumor recurrences, $2(15 \%)$ of 13 patients with both local and distant tumor recurrence, and $15(22 \%)$ of 68 patients with isolated distant recurrences had no prior history of WBRT. The median time to imaging-verified disease progression was 2.1 months (range 1-51 months). Six-month and 1-year estimated progression-free survivals were $25 \%$ and $13 \%$, respectively. Kaplan-Meier curves for overall and progression-free survivals are shown in Fig. 1.

Multivariate analysis revealed that several factors were significant independent predictors of poor prognosis (Table 2): 1) lower KPS score at the time of treatment ( $\mathrm{p}<$ $0.0001)$; 2) concurrent extracranial metastases to multiple organs other than the brain ( $\mathrm{p}=0.0001) ; 3)$ patient age $\geq$ 60 years $(p=0.004)$; and 4$)$ greater intracranial burden of disease ( $\mathrm{p}=0.03$ ) (Fig. 2).

After GKS had been performed for $\geq 5$ brain metastases, salvage treatments were performed in 57 patients (34\%). Whole-brain radiation therapy was performed af-

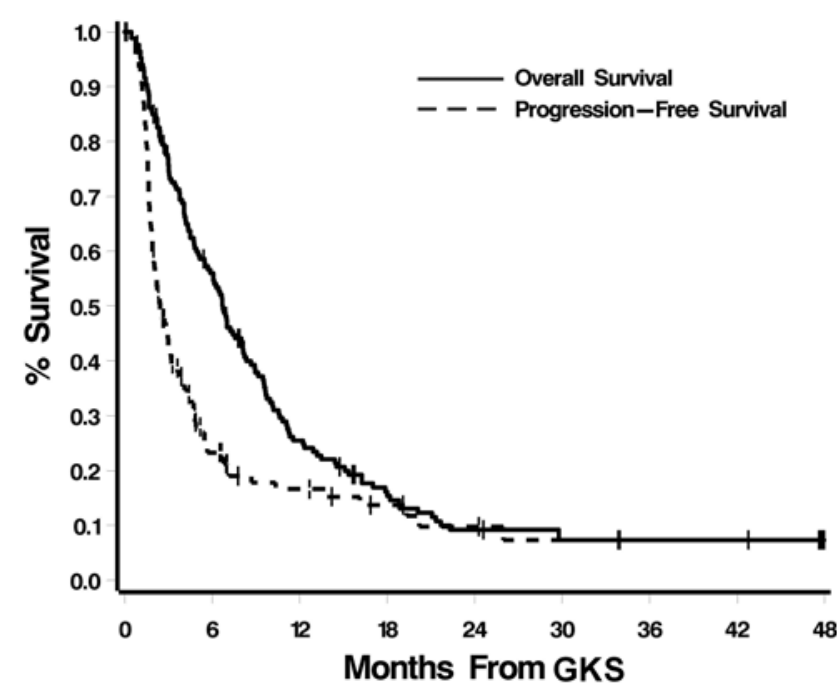
als.

FIG. 1. Kaplan-Meier curves for overall and progression-free surviv-

ter GKS in 28 patients $(16 \%)$ without a prior history of WBRT. Salvage GKS was performed alone or in combination with WBRT in 38 patients (22\%), and salvage surgery was performed in 5 patients (3\%). Intralesional hemorrhage after GKS occurred in 4 patients (2\%), all of whom were treated conservatively with expectant management and none of whom required any surgical intervention. Presumed radiation necrosis (diagnosis based on findings on MR perfusion studies or PET scan characteristics) occurred in 9 patients (5\%). There was no GKSrelated mortality in the patients in the study group.

\section{Discussion}

Whole-brain radiation therapy has been considered the primary treatment modality in patients with $\geq$ 5 brain metastases. ${ }^{40}$ Given that WBRT is typically performed only once and that most patients with $\geq 5$ brain metastases are patients who have recurrent intracranial disease (64\% in our series), a large number of these patients will already have received WBRT as part of their primary treatment (54\% in our series). If radiosurgery is not offered as an additive treatment, supportive medical management is usually the only remaining option and is associated with a survival time of 1-2 months. ${ }^{15,41,44}$ In our series, the median survival time for patients with tumor recurrence after prior WBRT was 7.1 months, which is a better survival time than those reported historically after medical management.

For patients without prior WBRT, the role of upfront WBRT as part of the treatment paradigm for multiple brain metastases is still debated. In 2011, Tsao et al.$^{38}$ performed a meta-analysis of RCTs evaluating radiosurgery, WBRT, and combination treatment for patients with $\leq 4$ brain metastases. Two RCTs comparing WBRT alone with WBRT plus a radiosurgery boost, ${ }^{2,21}$ and three RCTs comparing radiosurgery alone with radiosurgery plus WBRT, $3,7,20$ were evaluated. In two RCTs ${ }^{2,21}$ comparing WBRT alone with WBRT plus radiosurgery, no survival benefit was observed in patients with multiple (2-4) brain metastases 


\section{Radiosurgery for $\geq 5$ brain metastases}

TABLE 2: Results of the multivariate analysis for overall survival after GKS for $\geq 5$ metastases

\begin{tabular}{|c|c|c|c|}
\hline Factor & Better Prognostic Group & $\mathrm{HR}(95 \% \mathrm{Cl})$ & $\mathrm{p}$ Value \\
\hline $\begin{array}{l}\text { KPS score at time of GKS (60 vs } 70 \text { vs } \\
80 \text { vs } 90-100)\end{array}$ & higher KPS & $1.56(1.25-1.91)$ & $<0.0001$ \\
\hline $\begin{array}{l}\text { concurrent extracranial metastases ( } \geq 2 \\
\text { vs } 0-1 \text { metastases) }\end{array}$ & $0-1$ metastasis & $1.94(1.38-2.74)$ & 0.0001 \\
\hline patient age at GKS (>60 yrs vs $\leq 60 \mathrm{yrs}$ ) & $\leq 60$ yrs & $1.65(1.16-2.36)$ & 0.004 \\
\hline $\begin{array}{l}\text { total intracranial disease burden }(>10 \\
\left.\mathrm{cm}^{3} \text { vs } 1-10 \mathrm{~cm}^{3} \text { vs }<1 \mathrm{~cm}^{3}\right)\end{array}$ & smaller burden & $1.39(1.04-1.86)$ & 0.03 \\
\hline
\end{tabular}

when radiosurgery was used as a boost to WBRT (HR 1.63; 95\% CI 0.72-3.69; $\mathrm{p}=0$ 0.24). For patients with a single brain metastasis, one study demonstrated a statistically significant improvement in overall survival when a radiosurgery boost was added to WBRT (median survival time 6.5 months vs 4.9 months, $\mathrm{p}=0.03) .^{2}$ On the other hand, all three $\mathrm{RCTs}^{3,7,20}$ that evaluated radiosurgery versus WBRT plus radiosurgery reported that no survival benefit was obtained by adding WBRT to radiosurgery in patients with 1-4 brain metastases. Pooled data from two studies (Aoyama and Chang and their colleagues) ${ }^{3,7}$ again showed no survival benefit obtained by adding WBRT to radiosurgery (HR 0.98; 95\% CI 0.71-1.35, p=0.88). Both local and distant tumor control rates were much better when a combination of radiosurgery and WBRT was given to all patients; however, no significant difference in neurological cause of death was found when the combined treat-
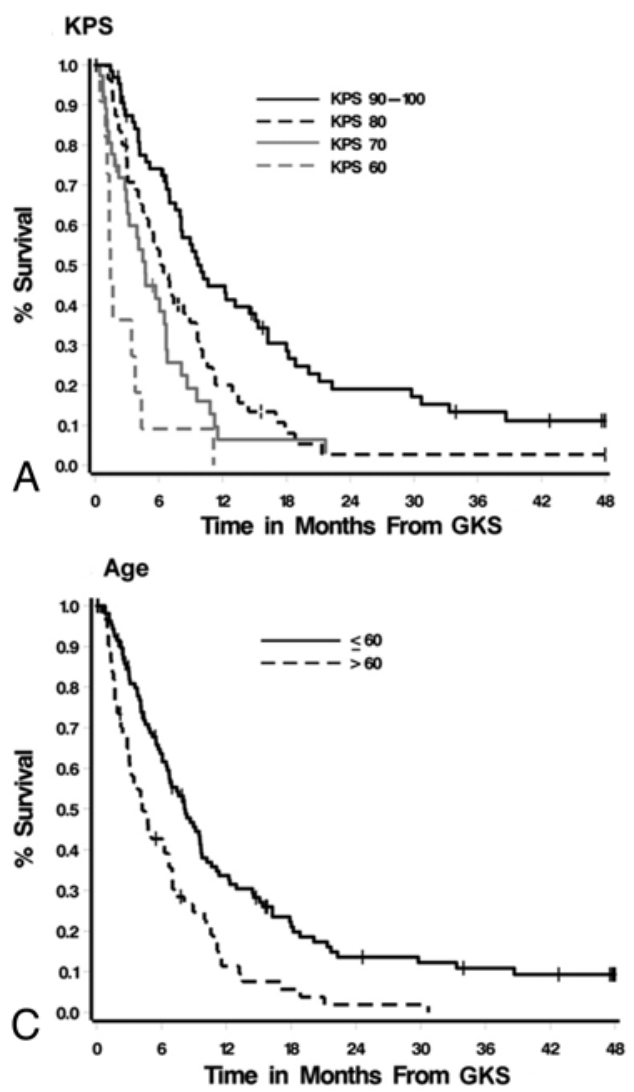

ment was compared with WBRT or radiosurgery alone..$^{38}$ In addition, Chang et al. ${ }^{7}$ reported better neurocognitive outcomes in patients with a limited number $(\leq 3)$ of brain metastases when radiosurgery alone was used without adjuvant WBRT.

For patients with $\geq 5$ brain metastases, there is no Level I evidence comparing the impact of WBRT versus radiosurgery on overall survival. Current evidence is limited to a few retrospective studies in which overall survival after radiosurgery was evaluated., 4, 18,22,28,32,43 Specifically, findings of most retrospective studies have not revealed a survival difference when patients with higher numbers of metastases are treated. Serizawa et al. ${ }^{28}$ performed a retrospective study of 778 patients with 1-10 brain metastases who were treated with SRS and concluded that the number of brain tumors has no effect on survival, whereas the extracranial disease burden, lower
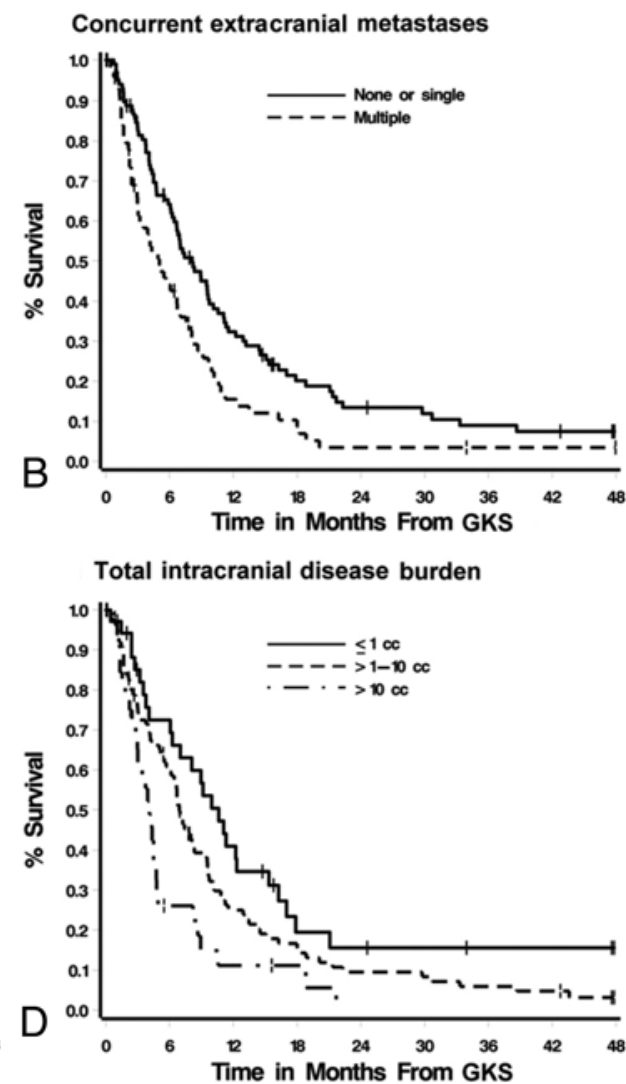

FIG. 2. Kaplan-Meier curves for overall survival of statistically significant independent prognostic factors at the time of GKS for $\geq 5$ brain metastases. 
KPS score, and male sex are factors associated with a poor prognosis. Bhatnagar and colleagues ${ }^{4}$ reviewed the outcomes of 205 patients with $\geq 4$ metastatic brain lesions treated with radiosurgery and found no difference in survival as the number of lesions increased. However, they did find that the total intracranial tumor volume, patient age, and RPA class were significant prognostic factors. Karlsson et al. ${ }^{18}$ reported a series of 1885 patients with brain metastases (1-8 lesions) treated with SRS over a 30 -year period. Those authors found no survival difference as the number of lesions increased in patients with $>2$ metastases, while control of the primary tumor was significantly associated with improved survival. Other groups have similarly found no relation between the number of brain metastases and survival. ${ }^{8,22,32}$ Furthermore, Suzuki et al. ${ }^{36}$ and Kim and colleagues ${ }^{19}$ reported relatively favorable survival outcomes and complication rates in patients with $>10$ brain metastases treated with SRS. In contrast, Yamamoto et al ${ }^{43}$ reported a series of 1676 patients with 1-85 brain metastases and concluded that the number of lesions did have a statistically significant impact on survival. However, $85 \%$ of their patients died of nonneurological causes, which highlights the fact that most patients die of systemic disease progression rather than neurological disease progression.

Current evidence suggests that WBRT may not be the sole therapeutic option for patients with $\geq 5$ brain metastases. First, in patients with a limited number of brain metastases ( $\leq 4)$, there is Level 1 evidence that no survival benefit is conferred by addition of WBRT to upfront radiosurgery. ${ }^{3,720}$ Furthermore, in patients with a single brain metastasis, the addition of SRS to WBRT confers a survival benefit. ${ }^{2}$ Second, multiple retrospective studies have revealed no significant survival difference in patients with multiple brain metastases as the number of metastases increases. , $, 8,18,22,28,32$ Hence, either SRS alone or a SRS boost to WBRT may be a reasonable option for upfront treatment of patients with $\geq 5$ brain metastases who have no previous history of WBRT. In addition, postponing WBRT may be of particular importance in preventing neurocognitive decline in patients with a clinical profile predictive of longer survival (the median survival time in 30 patients with $0-1$ poor prognostic factors was 16.3 months in our study). ${ }^{7,10}$

In 1997, Gaspar et al. ${ }^{12}$ performed an RPA on 1200 patients with brain metastases from 3 Radiation Therapy Oncology Group trials and determined 3 different classes based on patient age, KPS score, and systemic disease status. Although the analysis was performed in patients who were treated with WBRT as the initial therapy, the same prognostic factors have also been shown to be predictive in most studies in which SRS was used as a treatment modality. ${ }^{1-5,18-23,25,28,29,33,35}$ For patients with multiple lesions $(\geq 4)$, Bhatnagar et al. ${ }^{5}$ performed an RPA on a series of 205 patients and found that the total intracranial burden of disease was an important prognostic factor for patients with multiple brain metastases. In our series a lower KPS score at the time of treatment, age $>60$ years, multiple extracranial metastases, and greater total intracranial burden of disease were independent predictors of poorer survival after GKS for $\geq 5$ lesions.
Our series shows that GKS is a valuable, effective, and well-tolerated treatment modality for patients with $\geq 5$ intracranial lesions. In this group of patients, especially those with favorable prognostic factors, GKS can be offered (with or without WBRT, depending on prior treatment and clinical decision making) for treatment of intracranial metastases irrespective of the number of presenting lesions.

There were some limitations in our study. First, we had a heterogeneous group of patients with upfront and salvage treatments and different diseases. Wherever possible we have tried to eliminate these confounding effects by using appropriate statistical methods. Of note, we did not find any significant influence of most of them (for example WBRT) in our final results (Table 1). Also, despite the fact that our inclusion/exclusion criteria were designed liberally to include almost all patients who underwent GKS for $\geq 5$ brain metastases, this design cannot completely eliminate the selection bias that is common in nonrandomized studies nor account for evolving management strategies over time. Hence, there may be a bias in selecting patients with better prognoses for GKS, a feature acknowledged in our final conclusion. Third, the presumed advantage of GKS in preserving neurocognitive function has not been addressed in this study, and sufficient information is lacking in this series to draw any conclusions regarding this in the patient cohort. Further, no recommendations can be made regarding the upper limit of metastases appropriate for treatment with GKS. These features were beyond the scope of the current study and need to be addressed in future efforts. Finally, the cost-effectiveness of the more expensive GKS (versus WBRT) compared with its potential benefits as an upfront therapy in patients with $\geq 5$ brain metastases should be evaluated in a controlled prospective study.

\section{Conclusions}

In this study, GKS was a safe and effective upfront and salvage treatment for patients with $\geq 5$ brain metastases. It may be used as the sole treatment modality instead of WBRT, or it can be offered in combination with upfront WBRT. Patients with a favorable clinical prognostic profile may particularly benefit from GKS as part of their treatment regimen. Future controlled randomized trials are needed to further clarify the role of GKS in patients with a high number of brain metastases.

\section{Disclosure}

This work was supported in part by a grant from the American Association of Neurological Surgeons William P. VanWagenen Fellowship Program (N.F.M.), by Grant No. W81XWH-062-0033 from the US Department of Defense Breast Cancer Research Program (R.J.W.) and by the Melvin Burkhardt Chair in Neurosurgical Oncology and the Karen Colina Wilson research endowment within the Brain Tumor and Neuro-Oncology Center at the Cleveland Clinic Foundation (R.J.W.).

Dr. Neyman is a consultant for Elekta AB. The other authors report no potential conflict of interest concerning the materials or methods used in this study or the findings specified in this paper, but in the interest of full disclosure we declare the following: Dr. Recinos is a consultant for Vycor Medical, Inc., and has received 
an educational honorarium from Stryker; Dr. Suh is a consultant for Abbott Labs; Dr. Vogelbaum has received honoraria from Merck, Astra-Zeneca, and Schering-Plough; and Dr. Barnett is a consultant for Monteris Medical, Inc.

Author contributions to the study and manuscript preparation include the following. Conception and design: all authors. Acquisition of data: Mohammadi, Neyman. Analysis and interpretation of data: Mohammadi, Marko, Elson. Drafting the article: Mohammadi, Recinos. Critically revising the article: all authors. Reviewed submitted version of manuscript: all authors. Approved the final version of the manuscript on behalf of all authors: Angelov. Statistical analysis: Marko, Elson. Administrative/technical/material support: Neyman. Study supervision: Angelov, Mohammadi, Barnett, Weil, Vogelbaum, Chao, Suh, Neyman.

\section{References}

1. Alexander E III, Moriarty TM, Davis RB, Wen PY, Fine HA, Black PM, et al: Stereotactic radiosurgery for the definitive, noninvasive treatment of brain metastases. J Natl Cancer Inst 87:34-40, 1995

2. Andrews DW, Scott CB, Sperduto PW, Flanders AE, Gaspar LE, Schell MC, et al: Whole brain radiation therapy with or without stereotactic radiosurgery boost for patients with one to three brain metastases: phase III results of the RTOG 9508 randomised trial. Lancet 363:1665-1672, 2004

3. Aoyama H, Shirato H, Tago M, Nakagawa K, Toyoda T, Hatano $\mathrm{K}$, et al: Stereotactic radiosurgery plus whole-brain radiation therapy vs stereotactic radiosurgery alone for treatment of brain metastases: a randomized controlled trial. JAMA 295:2483-2491, 2006

4. Bhatnagar AK, Flickinger JC, Kondziolka D, Lunsford LD: Stereotactic radiosurgery for four or more intracranial metastases. Int J Radiat Oncol Biol Phys 64:898-903, 2006

5. Bhatnagar AK, Kondziolka D, Lunsford LD, Flickinger JC: Recursive partitioning analysis of prognostic factors for patients with four or more intracranial metastases treated with radiosurgery. Technol Cancer Res Treat 6:153-160, 2007

6. Cairncross JG, Kim JH, Posner JB: Radiation therapy for brain metastases. Ann Neurol 7:529-541, 1980

7. Chang EL, Wefel JS, Hess KR, Allen PK, Lang FF, Kornguth DG, et al: Neurocognition in patients with brain metastases treated with radiosurgery or radiosurgery plus whole-brain irradiation: a randomised controlled trial. Lancet Oncol 10: 1037-1044, 2009

8. Chang WS, Kim HY, Chang JW, Park YG, Chang JH: Analysis of radiosurgical results in patients with brain metastases according to the number of brain lesions: is stereotactic radiosurgery effective for multiple brain metastases? Clinical article. J Neurosurg 113 Suppl:73-78, 2010

9. Coia LR, Aaronson N, Linggood R, Loeffler J, Priestman TJ: A report of the consensus workshop panel on the treatment of brain metastases. Int J Radiat Oncol Biol Phys 23:223-227, 1992

10. DeAngelis LM, Delattre JY, Posner JB: Radiation-induced dementia in patients cured of brain metastases. Neurology 39:789-796, 1989

11. Elaimy AL, Mackay AR, Lamoreaux WT, Fairbanks RK, Demakas JJ, Cooke BS, et al: Clinical outcomes of stereotactic radiosurgery in the treatment of patients with metastatic brain tumors. World Neurosurg 75:673-683, 2011

12. Gaspar L, Scott C, Rotman M, Asbell S, Phillips T, Wasserman T, et al: Recursive partitioning analysis (RPA) of prognostic factors in three Radiation Therapy Oncology Group (RTOG) brain metastases trials. Int J Radiat Oncol Biol Phys 37:745-751, 1997

13. Gaspar LE, Scott C, Murray K, Curran W: Validation of the RTOG recursive partitioning analysis (RPA) classification for brain metastases. Int J Radiat Oncol Biol Phys 47:10011006,2000
14. Hazard LJ, Jensen RL, Shrieve DC: Role of stereotactic radiosurgery in the treatment of brain metastases. Am J Clin Oncol 28:403-410, 2005

15. Horton J, Baxter DH, Olson KB: The management of metastases to the brain by irradiation and corticosteroids. Am J Roentgenol Radium Ther Nucl Med 111:334-336, 1971

16. Hunter GK, Suh JH, Reuther AM, Vogelbaum MA, Barnett GH, Angelov L, et al: Treatment of five or more brain metastases with stereotactic radiosurgery. Int J Radiat Oncol Biol Phys 83:1394-1398, 2012

17. Joseph J, Adler JR, Cox RS, Hancock SL: Linear acceleratorbased stereotaxic radiosurgery for brain metastases: the influence of number of lesions on survival. J Clin Oncol 14:10851092, 1996

18. Karlsson B, Hanssens P, Wolff R, Söderman M, Lindquist C, Beute G: Thirty years' experience with Gamma Knife surgery for metastases to the brain. Clinical article. J Neurosurg 111: 449-457, 2009

19. Kim CH, Im YS, Nam DH, Park K, Kim JH, Lee JI: Gamma knife radiosurgery for ten or more brain metastases. J Korean Neurosurg Soc 44:358-363, 2008

20. Kocher M, Soffietti R, Abacioglu U, Villà S, Fauchon F, Baumert BG, et al: Adjuvant whole-brain radiotherapy versus observation after radiosurgery or surgical resection of one to three cerebral metastases: results of the EORTC 22952-26001 study. J Clin Oncol 29:134-141, 2011

21. Kondziolka D, Patel A, Lunsford LD, Kassam A, Flickinger JC: Stereotactic radiosurgery plus whole brain radiotherapy versus radiotherapy alone for patients with multiple brain metastases. Int J Radiat Oncol Biol Phys 45:427-434, 1999

22. Nam TK, Lee JI, Jung YJ, Im YS, An HY, Nam DH, et al: Gamma knife surgery for brain metastases in patients harboring four or more lesions: survival and prognostic factors. J Neurosurg 102 Suppl:147-150, 2005

23. Pan HC, Sun MH, Chen CCC, Chen CJ, Lee CH, Sheehan J: Neuroimaging and quality-of-life outcomes in patients with brain metastasis and peritumoral edema who undergo Gamma Knife surgery. J Neurosurg 109 Suppl:90-98, 2008

24. Patchell RA: The management of brain metastases. Cancer Treat Rev 29:533-540, 2003

25. Pollock BE, Brown PD, Foote RL, Stafford SL, Schomberg PJ: Properly selected patients with multiple brain metastases may benefit from aggressive treatment of their intracranial disease. J Neurooncol 61:73-80, 2003

26. Posner JB, Chernik NL: Intracranial metastases from systemic cancer. Adv Neurol 19:579-592, 1978

27. Regine WF, Scott C, Murray K, Curran W: Neurocognitive outcome in brain metastases patients treated with acceleratedfractionation vs. accelerated-hyperfractionated radiotherapy: an analysis from Radiation Therapy Oncology Group Study 91-04. Int J Radiat Oncol Biol Phys 51:711-717, 2001

28. Serizawa T, Hirai T, Nagano O, Higuchi Y, Matsuda S, Ono J, et al: Gamma knife surgery for 1-10 brain metastases without prophylactic whole-brain radiation therapy: analysis of cases meeting the Japanese prospective multi-institute study (JLGK0901) inclusion criteria. J Neurooncol 98:163-167, 2010

29. Serizawa T, Yamamoto M, Sato Y, Higuchi Y, Nagano O, Kawabe T, et al: Gamma Knife surgery as sole treatment for multiple brain metastases: 2-center retrospective review of 1508 cases meeting the inclusion criteria of the JLGK0901 multi-institutional prospective study. Clinical article. J Neurosurg 113 Suppl:48-52, 2010

30. Shaw E, Scott C, Souhami L, Dinapoli R, Kline R, Loeffler J, et al: Single dose radiosurgical treatment of recurrent previously irradiated primary brain tumors and brain metastases: final report of RTOG protocol 90-05. Int J Radiat Oncol Biol Phys 47:291-298, 2000

31. Shiau CY, Sneed PK, Shu HK, Lamborn KR, McDermott MW, Chang S, et al: Radiosurgery for brain metastases: rela- 
tionship of dose and pattern of enhancement to local control. Int J Radiat Oncol Biol Phys 37:375-383, 1997

32. Shuto T, Fujino H, Inomori S, Nagano H: Repeated gamma knife radiosurgery for multiple metastatic brain tumours. Acta Neurochir (Wien) 146:989-993, 2004

33. Skeie BS, Skeie GO, Enger PØ, Ganz JC, Heggdal JI, Ystevik $\mathrm{B}$, et al: Gamma knife surgery in brain melanomas: absence of extracranial metastases and tumor volume strongest indicators of prolonged survival. World Neurosurg 75:684-603, 2010

34. Soffietti R, Rudā R, Mutani R: Management of brain metastases. J Neurol 249:1357-1369, 2002

35. Sperduto CM, Watanabe Y, Mullan J, Hood T, Dyste G, Watts $\mathrm{C}$, et al: A validation study of a new prognostic index for patients with brain metastases: the Graded Prognostic Assessment. J Neurosurg 109 Suppl:87-89, 2008

36. Suzuki S, Omagari J, Nishio S, Nishiye E, Fukui M: Gamma knife radiosurgery for simultaneous multiple metastatic brain tumors. J Neurosurg 93 Suppl 3:30-31, 2000

37. Tosoni A, Ermani M, Brandes AA: The pathogenesis and treatment of brain metastases: a comprehensive review. Crit Rev Oncol Hematol 52:199-215, 2004

38. Tsao M, Xu W, Sahgal A: A meta-analysis evaluating stereotactic radiosurgery, whole-brain radiotherapy, or both for patients presenting with a limited number of brain metastases. Cancer 118:2486-2493, 2012

39. Tsao MN, Lloyd N, Wong R, Chow E, Rakovitch E, Laperriere $\mathrm{N}$ : Whole brain radiotherapy for the treatment of multiple brain metastases. Cochrane Database Syst Rev 3:CD003869, 2006

40. Tsao MN, Lloyd NS, Wong RK: Clinical practice guideline on the optimal radiotherapeutic management of brain metastases. BMC Cancer 5:34, 2005

41. Tsao MN, Lloyd NS, Wong RK, Rakovitch E, Chow E, Laperriere N: Radiotherapeutic management of brain metastases: a systematic review and meta-analysis. Cancer Treat Rev 31: 256-273, 2005

42. Vecht CJ, Haaxma-Reiche H, Noordijk EM, Padberg GW, Voormolen JH, Hoekstra FH, et al: Treatment of single brain metastasis: radiotherapy alone or combined with neurosurgery? Ann Neurol 33:583-590, 1993

43. Yamamoto M, Kawabe T, Barfod BE: How many metastases can be treated with radiosurgery? Prog Neurol Surg 25:261272,2012

44. Zimm S, Wampler GL, Stablein D, Hazra T, Young HF: Intracerebral metastases in solid-tumor patients: natural history and results of treatment. Cancer 48:384-394, 1981

Manuscript submitted May 15, 2012.

Accepted August 1, 2012.

This paper was presented as a platform presentation at the 16th International Leksell Gamma Knife Society meeting, Sydney, Australia, March 25-29, 2012.

Please include this information when citing this paper: DOI: 10.3171/2012.8.GKS12983.

Address correspondence to: Lilyana Angelov, M.D., Cleveland Clinic, 9500 Euclid Avenue, Cleveland, Ohio 44195. email: angelol @ ccf.org. 